\title{
Computational Study of Three Numerical Methods for Some Linear and Non-Linear Advection-Diffusion-Reaction Problems
}

\author{
A.R. Appadu ${ }^{1}$, J.M.-S Lubuma ${ }^{2}$ and N. Mphephu ${ }^{3}$ \\ 1,2,3 Department of Mathematics and Applied Mathematics, University of Pretoria, Pretoria, 0002, \\ South Africa. \\ Corresponding author: ${ }^{1}$ Email: Rao.Appadu@up.ac.za or biprao2@yahoo.com, ${ }^{2}$ Email: \\ Jean.Lubuma@up.ac.za, ${ }^{3}$ Email: mphephu.ndivhuwo@yahoo.com.
}

\begin{abstract}
In this paper, we use three existing schemes namely, Upwind Forward Euler, Non-Standard Finite Difference (NSFD) and Unconditionally Positive Finite Difference (UPFD) schemes to solve two numerical experiments described by a linear and a non-linear advection-diffusion-reaction equation with constant coefficients. These equations model exponential travelling waves and biofilm growth on a medical implant respectively. We study the exact and numerical dissipative and dispersive properties of the three schemes for both problems. Moreover, $L_{1}$ error, dispersion and dissipation errors, at some values of temporal and spatial step sizes have been computed for the three schemes for both problems.
\end{abstract}

\section{Keywords:}

advection-diffusion-reaction, stability, dispersion, dissipation, finite difference.

\section{Introduction}

The advection-diffusion-reaction partial differential equation provides a very useful and important mathematical model in a wide range of applications in natural sciences and engineering [10]. These applications include the transport of air, adsorption of pollutants in soil, diffusion of neutrons, food processing, modelling of biological systems, modelling of semiconductors, oil reservoir flow transport and reaction of chemical species etc. In hydrology, equations of this type govern the fate and transport of reactive pollutants and biofilm-forming microbes in porous media. These equations are often used to predict and control the extent of contamination in ground water systems [7]. In many of these applications, the unknown variables in the governing partial differential equation represent physical quantities that cannot take negative values such as pollutants, population, concentration of chemical compounds [6]. The application of advection-diffusion-reaction (ADR) equation is classified into three processes [9]. The first process is called convection and is due to movement of materials from one region to another. The second process is called diffusion and is due to movement of materials from a region of high concentration to a region of lower concentration. The last process is called reaction and is due to decay, adsorption and reaction of substances with other components. Qualitatively all the three processes form the ADR model that describe how the disturbed quantity being studied in the medium changes under the influence of these processes [9].

In many applications, the governing partial differential equation is non-linear elliptic and the analytical solution is not easy to find [10]. The difficulties in obtaining analytical solution lead many researchers and engineers to resort to numerical methods for approximating the solution to the problem. These numerical methods have been active for about fifty years now and the development of new techniques still attracts more attention [9]. However, there are also some challenges when applying these numerical methods to approximate the solution to problems that are non-linear. One of the challenges is numerical instabilities [10]. For the ADR model dominated with diffusion, the standard finite difference methods (SFDM) and finite element method (FEM) produce satisfactory results while for problems dominated by convection, numerical instabilities like oscillations and numerical dispersion appear in the solution approximated by these methods [9]. These numerical instabilities can be overcomed for instance 
by applying the Non-Standard Finite Difference methods (NSFD). These methods are now playing an important role in the design of reliable numerical schemes in several areas of science and engineering [12].

In this paper, we focus on the application of standard and Non-Standard Finite Difference methods to solve the one dimensional advection-diffusion-reaction equation. This equation takes the form

$$
\frac{\partial u}{\partial t}+\frac{\partial}{\partial x}(a u)=\frac{\partial}{\partial x}\left(D \frac{\partial u}{\partial x}\right)+f(u)
$$

where $u(x, t)$ is the unknown quantity being investigated, $a(x, t)$ is the velocity of the medium (also called convective velocity), $D(x, t)$ is the diffusion coefficient and $f(u)$ is the reaction source. The novelty in this work in regard to work in [6] is that the stability of the schemes have been obtained and hence it allows a more effective way of comparing the performance of the three schemes. Also, dissipation and dispersion properties of the schemes are studied.

The paper is organised as follows. In sections 2 and 3, we describe the numerical experiments considered and how the quantification of errors is implemented. In section 4, we study the stability, consistency, spectral analysis of three schemes when used to discretise $u_{t}+u_{x}-u_{x x}=-u$ and present the numerical results in section 5. Sections 6 and 7 discuss the stability, consistency, spectral analysis of the three methods when applied to $u_{t}-\left(D u_{x}\right)_{x}=r u(1-u)$ and numerical results are presented. Section 8 highlights the salient features of the paper.

\section{Numerical Experiments}

We consider two problems. First, we consider the advection-diffusion-reaction equation for modelling the exponential travelling waves [6]. This equation is given by

$$
\frac{\partial u}{\partial t}+\frac{\partial u}{\partial x}-\frac{\partial^{2} u}{\partial x^{2}}=-u, \quad(x, t) \in[0,10] \times[0,0.85],
$$

subject to the following initial condition and boundary conditions :

$$
\begin{aligned}
u(x, 0) & =\mathrm{e}^{-x}, \quad 0 \leq x \leq 10, \\
u(0, t) & =\mathrm{e}^{t}, \quad 0 \leq t \leq 0.85, \text { and } \\
u_{x}(10, t) & =-u(10, t), \quad 0 \leq t \leq 0.85 .
\end{aligned}
$$

Eq.(1) together with its initial and boundary conditions has the exact solution given by

$$
u(x, t)=\mathrm{e}^{(t-x)} .
$$

The most realistic applications involving equation Eq.(1) are for complex systems and as a result there will be grid spacing limitation on the numerical method. Thus, if the grid spacing is not fine enough, the numerical scheme will not compute stable solutions. Different methods have been proposed increasing local grid refinement only in those regions where the solution is changing rapidly [15].

Secondly, we consider the advection-diffusion-reaction equation for modelling biofilm growth on a medical implant [6]. This equation is given by

$$
\frac{\partial u}{\partial t}-\frac{\partial}{\partial x}\left(D \frac{\partial u}{\partial x}\right)=r u(1-u), \quad(x, t) \in[0,1] \times[0,13],
$$


with initial and boundary conditions given by

$$
u(x, 0)= \begin{cases}0.7 \mathrm{e}^{-\sigma\left(x-x_{0}\right)^{2}}, & \left|x-x_{0}\right| \leq 0.06 \\ 0, & \text { otherwise }\end{cases}
$$

and

$$
\begin{array}{ll}
u(0, t)=0, & 0 \leq t \leq 13 \\
u(1, t)=0 & , \quad 0 \leq t \leq 13 .
\end{array}
$$

Eq.(3) together with the initial and boundary conditions have its exact solution described by a travelling wave like solution [13] which is given by

$$
u(x, t)=\frac{1}{\left[1+\left(\frac{1-\sqrt{u(x, 0)}}{\sqrt{u(x, 0)} \exp \left(\frac{1}{6 D} \sqrt{6 D r} x\right)}\right) \exp \left(-\frac{5}{6} r t+\frac{1}{6 D} \sqrt{6 D r} x\right)\right]^{2}} .
$$

We solve problem 2 described by Eq.(3) using the same parameters as in [6], namely $D=0.0002$, $x_{0}=0.5, r=0.05$ and $\sigma=80$.

\section{Quantification of Errors}

To quantify the errors from numerical results into dispersion and dissipation errors, we follow the work of Takacs [18]. We let $u_{m}$ represent the analytical solution and $v_{m}$ represent the numerical solution, at grid point, $m$. Then, the absolute error is given by

$$
E=\left|u_{m}-v_{m}\right| .
$$

The Total Mean Square Error is calculated as

$$
\begin{aligned}
& E_{T M S}=\frac{1}{N} \sum_{m=1}^{N}\left(u_{m}-v_{m}\right)^{2}, \text { or } \\
& E_{T M S}=\frac{1}{N} \sum_{m=1}^{N} u_{m}^{2}-\frac{2}{N} \sum_{m=1}^{N} u_{m} v_{m}+\frac{1}{N} \sum_{m=1}^{N} v_{m}^{2},
\end{aligned}
$$

where $N$ is the number of grid points.

The sample variances for the analytical and numerical solutions are respectively given by

$$
\begin{aligned}
& \delta^{2}(u)=\frac{1}{N} \sum_{m=1}^{N}\left(u_{m}-\bar{u}\right)^{2}, \text { and } \\
& \delta^{2}(v)=\frac{1}{N} \sum_{m=1}^{N}\left(v_{m}-\bar{v}\right)^{2}, \text { where } \bar{u} \text { and } \bar{v} \text { are the means of } u \text { and } v \text { respectively. }
\end{aligned}
$$

Expanding the sample variance, we obtain

$$
\begin{aligned}
& \delta^{2}(u)=\frac{1}{N} \sum_{m=1}^{N} u_{m}^{2}-\frac{2}{N} \sum_{m=1}^{N} u_{m} \bar{u}+\frac{1}{N} \sum_{m=1}^{N} \bar{u}^{2}, \\
& \delta^{2}(v)=\frac{1}{N} \sum_{m=1}^{N} v_{m}^{2}-\frac{2}{N} \sum_{m=1}^{N} v_{m} \bar{v}+\frac{1}{N} \sum_{m=1}^{N} \bar{v}^{2} .
\end{aligned}
$$


The Total Mean Square Error can be expressed as

$$
\begin{aligned}
E_{T M S} & =\frac{1}{N} \sum_{m=1}^{N}\left(u_{m}-\bar{u}\right)^{2}+\frac{1}{N} \sum_{m=1}^{N}\left(v_{m}-\bar{v}\right)^{2}+\frac{2}{N} \sum_{m=1}^{N} u_{m} \bar{u} \\
& +\frac{2}{N} \sum_{m=1}^{N} v_{m} \bar{v}-\frac{1}{N} \sum_{m=1}^{N}(\bar{u})^{2}-\frac{1}{N} \sum_{m=1}^{N}(\bar{v})^{2}-\frac{2}{N} \sum_{m=1}^{N} u_{m} v_{m} .
\end{aligned}
$$

or

$$
\begin{aligned}
E_{T M S} & =\delta^{2}(u)+\delta^{2}(v)+2\left(\frac{1}{N} \sum_{m=1}^{N} u_{m} \bar{u}\right)+2\left(\frac{1}{N} \sum_{m=1}^{N} v_{m} \bar{v}\right) \\
& -\frac{1}{N} \sum_{m=1}^{N}(\bar{u})^{2}-\frac{1}{N} \sum_{m=1}^{N}(\bar{v})^{2}-2\left(\frac{1}{N} \sum_{m=1}^{N} u_{m} v_{m}-\bar{u} \bar{v}\right)-2 \bar{u} \bar{v} .
\end{aligned}
$$

Further simplification gives

$$
E_{T M S}=\delta^{2}(u)+\delta^{2}(v)+(\bar{u}-\bar{v})^{2}-2 \operatorname{Cov}(u, v) .
$$

The correlation coefficient is defined as

$$
\rho=\frac{\operatorname{Cov}(u, v)}{\delta(u) \delta(v)}
$$

Hence, the covariance can be expressed as

$$
\operatorname{Cov}(u, v)=\rho \delta(u) \delta(v) .
$$

We thus have,

$$
\begin{aligned}
E_{T M S} & =\delta^{2}(u)+\delta^{2}(v)+(\bar{u}-\bar{v})^{2}-2 \rho \delta(u) \delta(v) \\
& =\delta^{2}(u)+\delta^{2}(v)+(\bar{u}-\bar{v})^{2}-2 \rho \delta(u) \delta(v)+2 \delta(u) \delta(v)-2 \delta(u) \delta(v) \\
& =\delta^{2}(u)+\delta^{2}(v)-2 \delta(u) \delta(v)+(\bar{u}-\bar{v})^{2}-2 \rho \delta(u) \delta(v)+2 \delta(u) \delta(v) \\
& =(\delta(u)-\delta(v))^{2}+(\bar{u}-\bar{v})^{2}+2(1-\rho) \delta(u) \delta(v) .
\end{aligned}
$$

Setting $\rho=1$, we obtain

$$
E_{T M S}=(\delta(u)-\delta(v))^{2}+(\bar{u}-\bar{v})^{2} .
$$

Hence the dispersion error is

$$
2(1-\rho) \delta(u) \delta(v)
$$

while the dissipation error is

$$
(\delta(u)-\delta(v))^{2}+(\bar{u}-\bar{v})^{2} .
$$

The total error is defined as the sum of the dispersion and dissipation errors [18].

The $L_{1}$ error is given by

$$
\text { L1error }=h \sum_{m=1}^{N}\left|u_{m}-v_{m}\right| .
$$

Some interesting applications of the concept of quantification of errors into dispersion and dissipation can be found in $[2,3,18]$. 


\section{Numerical solution of $u_{t}+u_{x}-u_{x x}=-u$}

In this section, we use three finite difference schemes to solve $u_{t}+u_{x}-u_{x x}=-u$ subject to the specified boundary conditions, as detailed in section 2 .

\subsection{Upwind Forward Euler Finite Difference Scheme}

When Eq.(1) is discretised by the Upwind Forward Euler finite difference scheme [6], we get

$$
\frac{u_{m}^{n+1}-u_{m}^{n}}{k}+\frac{u_{m}^{n}-u_{m-1}^{n}}{h}-\frac{u_{m+1}^{n}-2 u_{m}^{n}+u_{m-1}^{n}}{h^{2}}=-u_{m}^{n},
$$

which can be rewritten as

$$
u_{m}^{n+1}=(1-k-\lambda-2 \beta) u_{m}^{n}+(\lambda+\beta) u_{m-1}^{n}+\beta u_{m+1}^{n},
$$

where $\lambda=\frac{k}{h}, \beta=\frac{k}{h^{2}}, k$ and $h$ are the temporal and spatial step sizes respectively.

\section{Stability}

To obtain the region of stability of the finite difference scheme, we use the Fourier series analysis. The amplification factor is given by

$$
\xi=1-k-(\lambda+2 \beta)(1-\cos \omega)-I \lambda \sin \omega,
$$

where $\omega$ is the phase angle, $\omega=\theta h$ where $\theta$ is the wavenumber and $h$ is the spatial step size.

The scheme is stable whenever the Von-Neumann condition, $|\xi| \leq 1$ is satisfied. The modulus of amplification factor is given by

$$
|\xi|=\sqrt{[\Re(\xi)]^{2}+[\Im(\xi)]^{2}}
$$

where $\Re(\xi)$ and $\Im(\xi)$ are the real and imaginary parts of $\xi$ respectively. The modulus of the amplification factor of the scheme given by Eq. (8) is obtained as

$$
|\xi|=\sqrt{\left[1-k-\left(\frac{k}{h}+\frac{2 k}{h^{2}}\right)[1-\cos (\omega)]\right]^{2}+\left[\frac{k \sin (\omega)}{h}\right]^{2}},
$$

with $-\pi \leq \omega \leq \pi$

We choose $h=0.1$ as in the numerical experiment we have worked with $h=0.1$. We thus get

$$
|\xi|^{2}=[1-k-210 k(1-\cos (\omega))]^{2}+(10 k \sin (\omega))^{2} .
$$

We then use maple software to solve for $|\xi|^{2} \leq 1$ for $\omega \in[-\pi, \pi]$ and we obtain the region of stability as $0<k \leq 0.00475$. 


\section{Consistency}

To study the consistency of the Upwind Forward Euler finite difference scheme, we need to first obtain the Taylor expansion of the terms in Eq.(8) about the point $(m, n)$. We assume that $u$ is continuous and that the derivatives of $u$ are bounded. The Taylor series expansion of $u_{m}^{n+1}, u_{m+1}^{n}$ and $u_{m-1}^{n}$ about the point $(m, n)$ are respectively given by

$$
\begin{aligned}
u_{m}^{n+1} & =u_{m}^{n}+k \frac{\partial u}{\partial t}+\frac{k^{2}}{2 !} \frac{\partial^{2} u}{\partial t^{2}}+\frac{k^{3}}{3 !} \frac{\partial^{3} u}{\partial t^{3}}+\cdots \\
u_{m+1}^{n} & =u_{m}^{n}+h \frac{\partial u}{\partial x}+\frac{h^{2}}{2 !} \frac{\partial^{2} u}{\partial x^{2}}+\frac{h^{3}}{3 !} \frac{\partial^{3} u}{\partial x^{3}}+\cdots \\
u_{m-1}^{n} & =u_{m}^{n}-h \frac{\partial u}{\partial x}+\frac{h^{2}}{2 !} \frac{\partial^{2} u}{\partial x^{2}}-\frac{h^{3}}{3 !} \frac{\partial^{3} u}{\partial x^{3}}+\cdots
\end{aligned}
$$

Substituting Eqs.(12)-(14) in Eq.(8) and simplifying, we obtain

$$
\frac{\partial u}{\partial t}+\frac{\partial u}{\partial x}-\frac{\partial^{2} u}{\partial x^{2}}=-u-\frac{k}{2} \frac{\partial^{2} u}{\partial t^{2}}-\frac{k^{2}}{6} \frac{\partial^{3} u}{\partial t^{3}}+\frac{h}{2} \frac{\partial^{2} u}{\partial x^{2}}-\frac{h^{2}}{6} \frac{\partial^{3} u}{\partial x^{3}}+\cdots
$$

As $k \rightarrow 0$ and $h \rightarrow 0$, Eq.(15) reduces to Eq.(1) and therefore, the Upwind Forward Euler finite difference scheme is consistent.

\section{Spectral Analysis}

We study the dissipative and dispersive behaviour of the Upwind Forward Euler finite difference scheme when $h=0.1$ at some different values of $k$. The values of $k$ were chosen such that they are less than or equal to the upper bound of the region of stability.

We let the elementary solution to Eq.(1) be [14]

$$
u(x, t)=\mathrm{e}^{\alpha t} \mathrm{e}^{I \theta x} .
$$

where $\alpha$ is the dispersion relation and $\theta$ is the wave number.

Using Eq.(16) and Eq.(1) and solving for the dispersion relation, $\alpha$ we obtain

$$
\alpha=-1-\theta^{2}-I \theta \text {. }
$$

As a result of Eq.(17), the elementary solution is now expressed as

$$
u(x, t)=\mathrm{e}^{\left(-1-\theta^{2}\right) t} \mathrm{e}^{-I \theta t} \mathrm{e}^{I \theta x} .
$$

The term $\mathrm{e}^{\left(-1-\theta^{2}\right) t} \mathrm{e}^{-I \theta t}$ shows that the amplitude of the solution is decaying exponentially and hence the exact amplification factor is given by

$$
\begin{aligned}
\xi_{\text {exact }} & =\frac{\mathrm{e}^{\left(-1-\theta^{2}\right)(n+1) k} \mathrm{e}^{-I \theta(n+1) k}}{\mathrm{e}^{\left(-1-\theta^{2}\right) n k} \mathrm{e}^{-I \theta n k}}, \\
& =\mathrm{e}^{\left(-1-\theta^{2}\right) k} \mathrm{e}^{-I \theta k} .
\end{aligned}
$$

Equivalently, the exact amplification factor can be expressed as

$$
\xi_{\text {exact }}=(\cos (\theta k)-I \sin (\theta k)) \mathrm{e}^{-\left(1+\theta^{2}\right) k} .
$$


The relative phase error, is given by

$$
\begin{aligned}
\mathrm{RPE} & =\frac{\arg \left(\xi_{\text {num }}\right)}{\arg \left(\xi_{\text {exact }}\right)}, \\
& =\frac{\arg \left(\xi_{\text {num }}\right)}{\tan ^{-1}(-\tan (\theta k))}, \\
& =-\frac{1}{k \theta} \arg \left(\xi_{\text {num }}\right), \\
& =-\frac{1}{k \theta} \tan ^{-1}\left(\frac{\Re\left(\xi_{\text {num }}\right)}{\Im\left(\xi_{\text {num }}\right)}\right) .
\end{aligned}
$$

where $\xi_{\text {num }}$ is the numerical amplification factor obtained from the Fourier series analysis. Thus the relative phase error for the Upwind Forward Euler scheme is given by

$$
\mathrm{RPE}=\frac{1}{k \theta} \tan ^{-1}\left(\frac{\lambda \sin \omega}{1-k-(\lambda+\beta)(1-\cos \omega)}\right) .
$$

We next obtain plots of the variation of the modulus of exact amplification factor (exact AFM) and modulus of the numerical amplification factor (numerical AFM), both versus phase angle at $h=0.1$ and at some different values of $k$ in Fig. (1). 


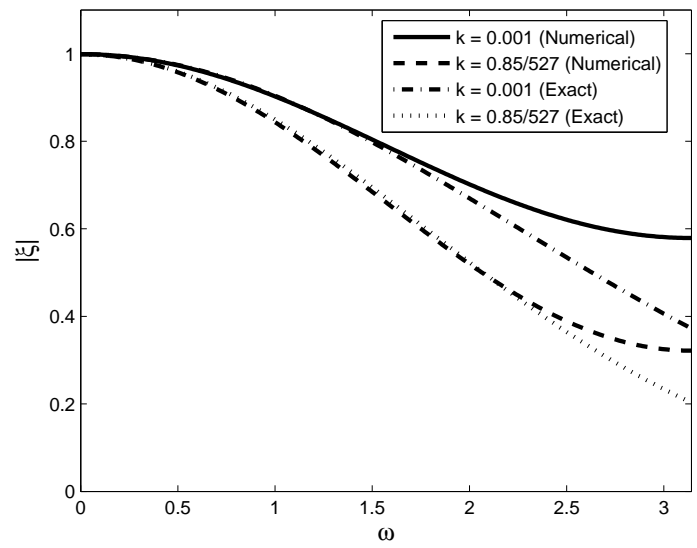

(a) $k=0.001$ and $k=\frac{0.85}{527}$

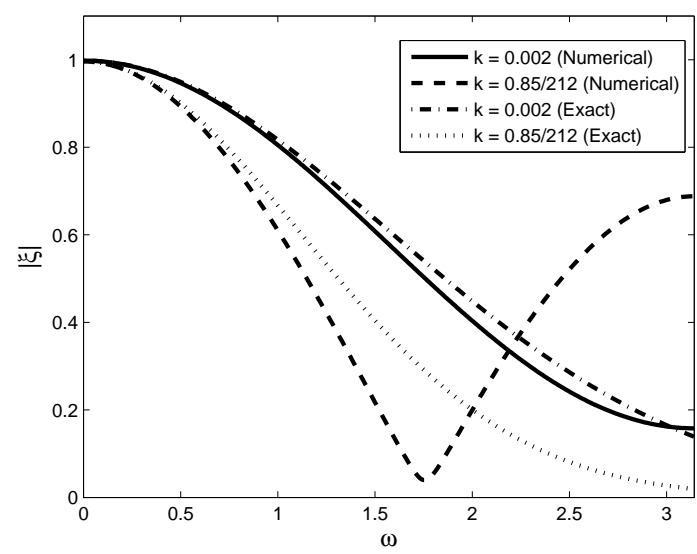

(b) $k=0.002$ and $k=\frac{0.85}{212}$

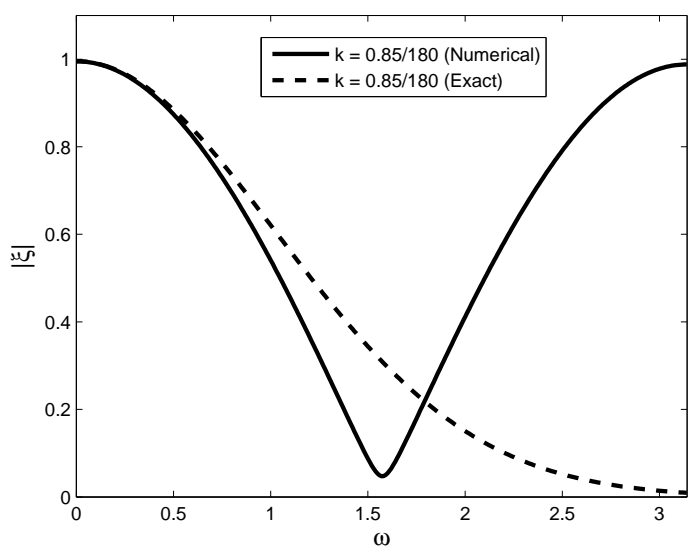

(c) $k=\frac{0.85}{180}$

Figure 1: Plots of the exact AFM and AFM for Upwind Forward Euler scheme for Eq.(1), at some values of $k$ with $h=0.1$. 


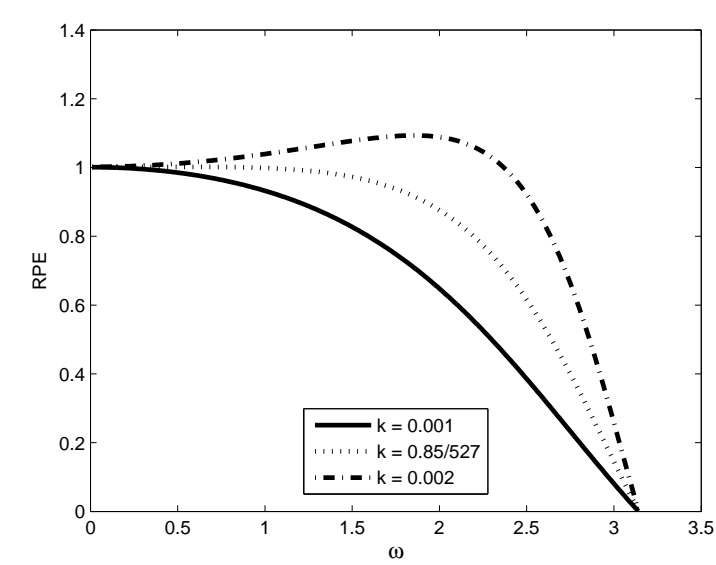

(a)

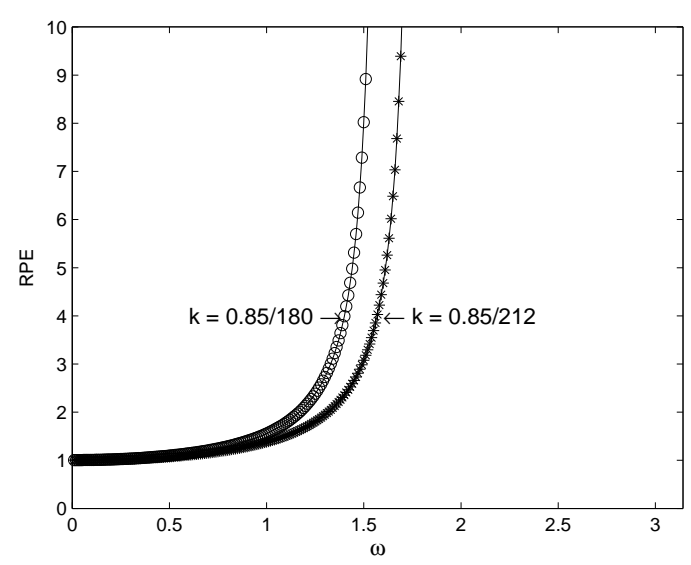

(b)

Figure 2: Plots of relative phase error vs phase angle for Upwind Forward Euler scheme discretising Eq.(1), at some values of $k$, with $h=0.1$.

From Fig. (1), we observe that at low phase angles, (i.e. 0 to 1 ), the curves of $\left|\xi_{\text {num }}\right|$ and $\left|\xi_{\text {exact }}\right|$ are very close to each other. In general, as the phase angle increases the numerical dissipation error increases. Figs.2(a) and 2(b) show that at $k=0.001$ and $k=\frac{0.85}{527}$, we have phase lag while at $k=\frac{0.85}{180}$ and $\frac{0.85}{212}$, we have phase lead for $\omega \in[0, \pi]$. At $k=0.002$, we have a phase lead for $\omega \in[0,2.36]$. The best dispersive property is obtained at $k=\frac{0.85}{527}$ for low phase angles.

\subsection{Non-Standard Finite Difference Scheme}

The Non-Standard Finite Difference scheme proposed by Mickens [11] for Eq. (1) is given by

$$
\frac{u_{m}^{n+1}-u_{m}^{n}}{k}+\frac{u_{m}^{n}-u_{m-1}^{n}}{h}-\frac{u_{m+1}^{n}-2 u_{m}^{n}+u_{m-1}^{n}}{h^{2}}=-u_{m}^{n+1},
$$

which simplifies as

$$
u_{m}^{n+1}=\left(\frac{1-\lambda-2 \beta}{1+k}\right) u_{m}^{n}+\left(\frac{\lambda+\beta}{1+k}\right) u_{m-1}^{n}+\left(\frac{\beta}{1+k}\right) u_{m+1}^{n},
$$

where $\lambda=\frac{k}{h}$ and $\beta=\frac{k}{h^{2}}$.

\section{Stability}

The amplification factor of the Non-Standard Finite Difference scheme is given by

$$
\xi=\frac{1-(\lambda+2 \beta)(1-\cos \omega)}{1+k}-I\left(\frac{\lambda \sin \omega}{1+k}\right),
$$

and the modulus of its amplification factor is calculated as

$$
|\xi|=\sqrt{\left[\frac{1-(\lambda+2 \beta)(1-\cos \omega)}{1+k}\right]^{2}+\left[\frac{\lambda \sin \omega}{1+k}\right]^{2}},
$$

with $\omega \in[-\pi, \pi]$. 
On plugging $h=0.1$, we get

$$
|\xi|^{2}=\frac{\left(1-210 k(1-\cos (\omega))^{2}+(10 k \sin (\omega))^{2}\right.}{(1+k)^{2}} .
$$

Solving $|\xi|^{2} \leq 1$ for $\omega \in[-\pi, \pi]$ gives the region of stability as $0<k \leq 0.00477$.

\section{Consistency}

To study the consistency of the Non-Standard Finite Difference scheme, we substitute Eqs.(12)-(14) in Eq. (23). Substituting these equations and simplifying, we obtain

$$
\begin{aligned}
\frac{\partial u}{\partial t}+\frac{\partial u}{\partial x}-\frac{\partial^{2} u}{\partial x^{2}} & =-u-k \frac{\partial u}{\partial t}+\left(\frac{k^{2}}{2}-\frac{k}{2}\right) \frac{\partial^{2} u}{\partial t^{2}}+\left(\frac{k^{3}}{6}-\frac{k^{2}}{6}\right) \frac{\partial^{3} u}{\partial t^{3}}+\frac{h}{2} \frac{\partial^{2} u}{\partial x^{2}} \\
& -\frac{h^{2}}{2} \frac{\partial^{3} u}{\partial x^{3}}+\cdots
\end{aligned}
$$

As $k \rightarrow 0$ and $h \rightarrow 0$, Eq.(27) reduces to Eq.(1), hence the scheme is consistent.

\section{Spectral Analysis}

We plot the variation of the modulus of the exact amplification factor and modulus of the numerical amplification factor and relative phase error with respect to the phase angle at some different values of $k$. The results are shown in Figs. (3) and (4).

Using Eqs.(21) and (24) with $h=0.1$, the relative phase error is calculated as

$$
\mathrm{RPE}=\frac{1}{k \theta} \tan ^{-1}\left(\frac{-10 k \sin \omega}{1+210 k(1-\cos \omega)}\right) .
$$

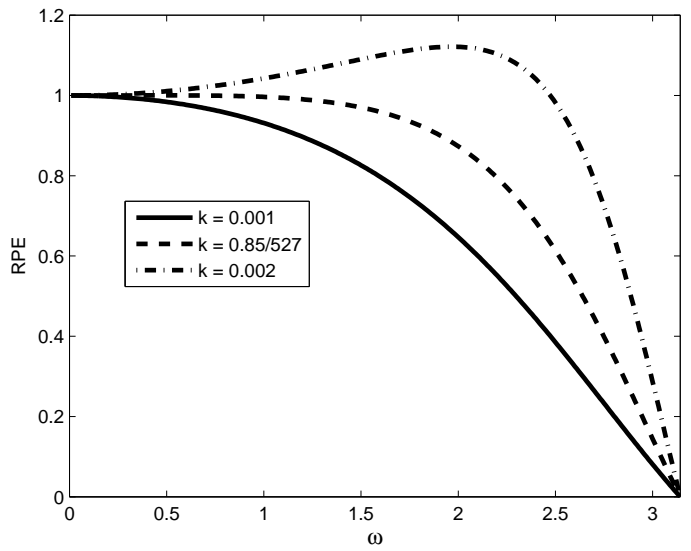

(a)

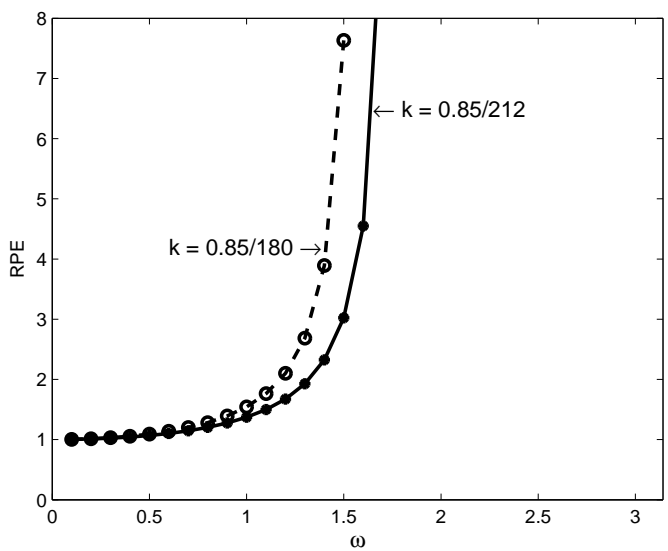

(b)

Figure 3: Plots of relative phase error vs phase angle for the Non-Standard Finite Difference Scheme discretising Eq.(1) at some values of $k$, with $h=0.1$. 


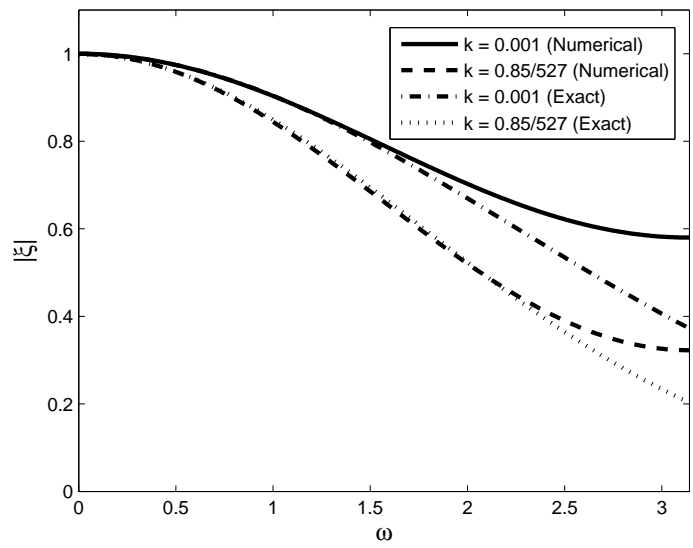

(a) $k=0.001$ and $k=\frac{0.85}{527}$

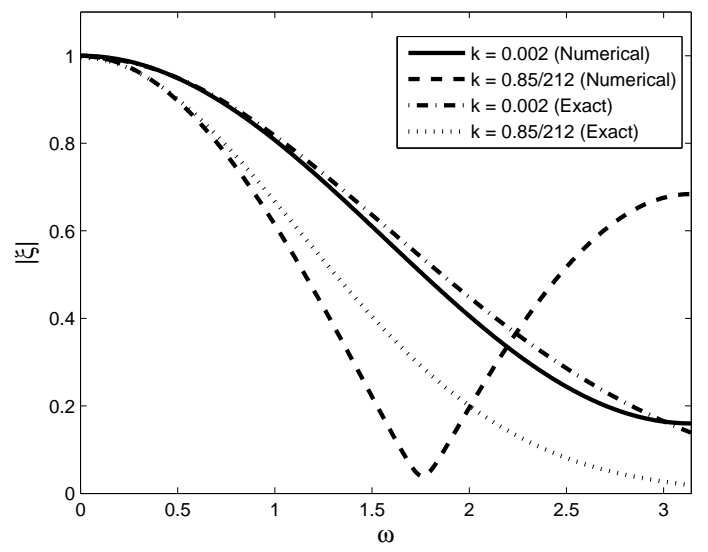

(b) $k=0.002$ and $k=\frac{0.85}{212}$

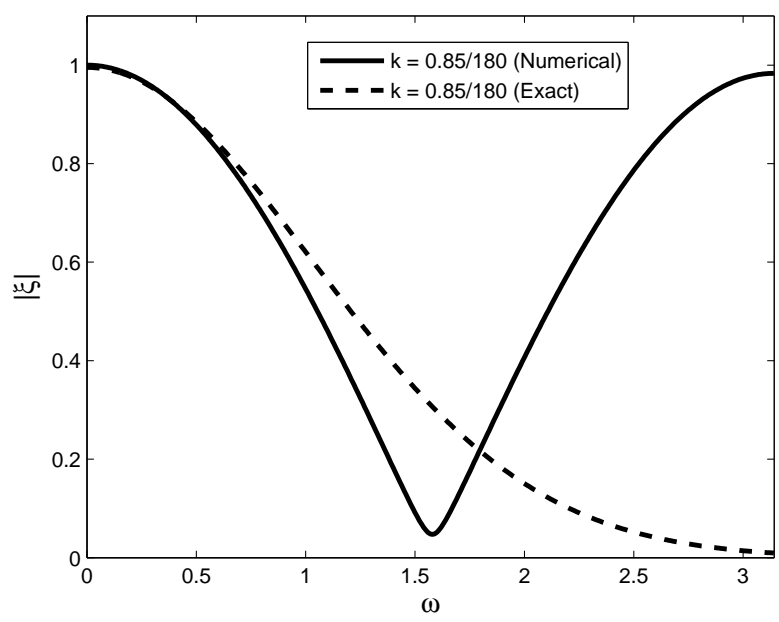

(c) $k=\frac{0.85}{180}$

Figure 4: Plot of the exact AFM and AFM of the Non-Standard Finite Difference scheme for Eq. (1), at some values of $k$, with $h=0.1$.

Based on Figs.(2) and (3), we observed that the dispersive behaviours of Non-Standard Finite Difference scheme is better than that of the Upwind Forward Euler scheme.

\subsection{Unconditionally Positive Finite Difference Scheme}

When discretised by the Unconditionally Positive Finite Difference Scheme [6], Eq.(1) gives

$$
\frac{u_{m}^{n+1}-u_{m}^{n}}{k}+\frac{u_{m}^{n+1}-u_{m-1}^{n}}{h}-\frac{u_{m+1}^{n}-2 u_{m}^{n+1}+u_{m-1}^{n}}{h^{2}}=-u_{m}^{n+1},
$$

which simplifies as

$$
u_{m}^{n+1}=\frac{u_{m}^{n}+(\lambda+\beta) u_{m-1}^{n}+\beta u_{m+1}^{n}}{1+k+\lambda+2 \beta},
$$


where $\lambda=\frac{k}{h}$ and $\beta=\frac{k}{h^{2}}$.

\section{Stability}

The amplification factor of the UPFD scheme is given by

$$
\xi=\frac{1+(\lambda+2 \beta) \cos \omega}{1+k+\lambda+2 \beta}-I\left[\frac{\lambda \sin \omega}{1+k+\lambda+2 \beta}\right] .
$$

The scheme is positive definite for all $k, h>0$. Hence, the scheme is unconditionally stable for all $h, k>0$.

\section{Consistency}

To obtain the truncation errors for the Unconditionally Positive Finite Difference method, we substitute Eqs.(12)-(14) in the finite difference scheme given by Eq.(29). Substituting these equations and simplifying, we obtain

$$
\begin{aligned}
\frac{\partial u}{\partial t}+\frac{\partial u}{\partial x}-\frac{\partial^{2} u}{\partial x^{2}} & =-u-\left(k+\frac{k}{h}+\frac{2 k}{h^{2}}\right) \frac{\partial u}{\partial t}-\left(\frac{k}{2}+\frac{k^{2}}{2}+\frac{k^{2}}{2 h}+\frac{k^{2}}{h^{2}}\right) \frac{\partial^{2} u}{\partial t^{2}} \\
& -\left(\frac{k^{2}}{6}+\frac{k^{3}}{6}+\frac{k^{3}}{6 h}+\frac{k^{3}}{3 h^{2}}\right) \frac{\partial^{3} u}{\partial t^{3}}+\frac{h}{2} \frac{\partial^{2} u}{\partial x^{2}}-\frac{h^{2}}{6} \frac{\partial^{3} u}{\partial x^{3}}+\cdots
\end{aligned}
$$

For the scheme to be consistent, we require $k=h^{3}$ which gives

$$
\begin{aligned}
\frac{\partial u}{\partial t}+\frac{\partial u}{\partial x}-\frac{\partial^{2} u}{\partial x^{2}} & =-u-\left(h^{3}+h^{2}+2 h\right) \frac{\partial u}{\partial t}-\frac{h^{3}}{2}\left(1+h^{2}+2 h+h^{3}\right) \frac{\partial^{2} u}{\partial t^{2}} \\
& -\frac{h^{6}}{6}\left(1+h^{2}+2 h+h^{3}\right) \frac{\partial^{3} u}{\partial t^{3}}+\frac{h}{2} \frac{\partial^{2} u}{\partial x^{2}}-\frac{h^{2}}{6} \frac{\partial^{3} u}{\partial x^{3}}+\cdots
\end{aligned}
$$

When $h \rightarrow 0$ we can see that Eq.(32) reduces to Eq.(1), hence the Unconditionally Positive Finite Difference Scheme is consistent when $k=h^{3}$.

\section{Spectral Analysis}

Using Eqs. (17) and (30) with $h=0.1$, the relative phase error is given by

$$
\mathrm{RPE}=\frac{1}{k \theta} \tan ^{-1}\left(\frac{-10 k \sin \omega}{1+210 k \cos \omega}\right) .
$$




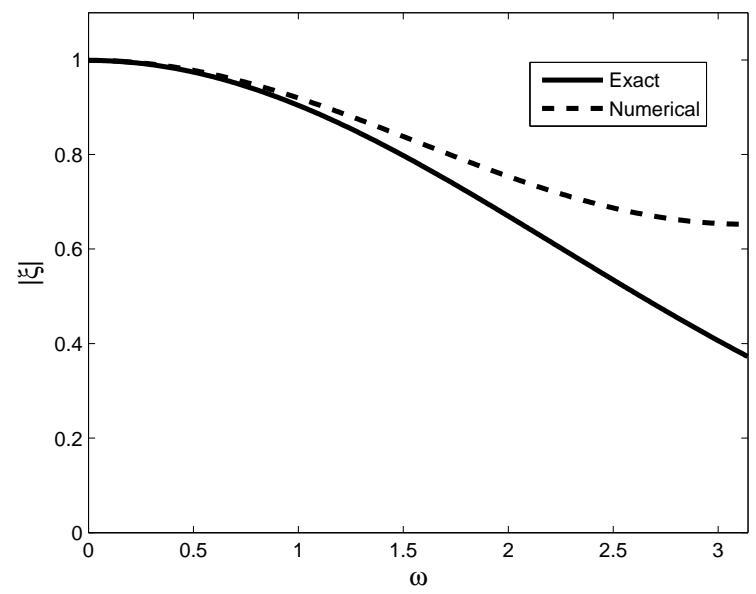

Figure 5: Plot of the exact AFM and AFM of the Unconditionally Positive Finite Difference scheme for Eq.(1) with $k=0.001$ and $h=0.1$.

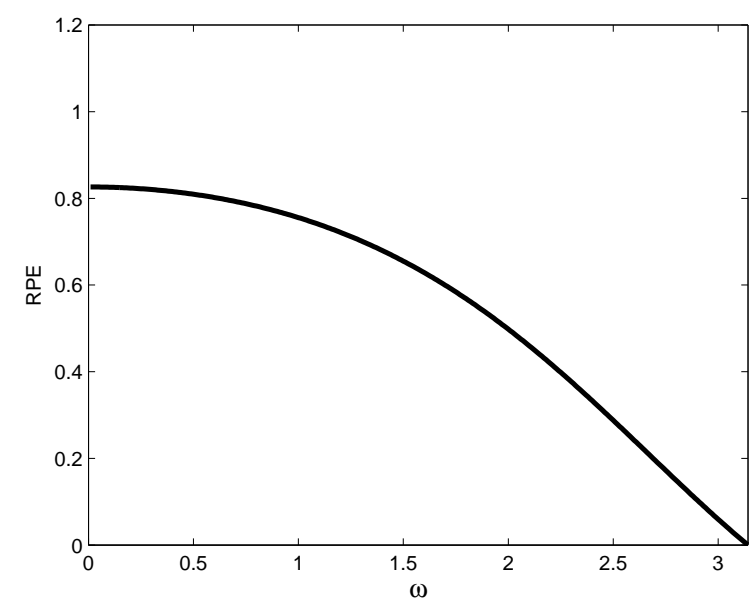

Figure 6: Plot of relative phase error vs phase angle for Unconditionally Positive Finite Difference Scheme discretising Eq.(1) with $k=0.001$ and $h=0.1$.

The plots of the modulus of amplification factor and relative phase error are shown in Figs. (5) and (6). We note that the relative phase error for the scheme is not one when $\omega=0$. This could be due to the presence of extra truncation error terms resulting from the approximation of first and second order derivative terms $\frac{\partial u}{\partial x}, \frac{\partial^{2} u}{\partial x^{2}}$ at different time levels. Also, we observe that the scheme is less dissipative than the partial differential equation as the phase angle increases.

\section{$5 \quad$ Numerical Results for Experiment 1}

We tabulate the error rate with respect to $L_{1}$ norm, dissipation, dispersion, and total errors in Tables (1) to (3) for the three numerical schemes.

We observe that for the Upwind Forward Euler and NSFD schemes, the errors are dependent on the temporal step size when $h$ is chosen as 0.1. The errors decrease as $k$ is increased, at a fixed $h$ which is 0.1 . Also, the dissipation error is slightly greater than the dispersion error for the five values of $k$ chosen for both schemes. 
From Figs. (2) and (4), we observe that as $k$ increases, the plots for numerical amplification factor deviate more from the exact amplification factor. However when we tabulate the results we observe that $E_{\text {num }}$, dissipation error, dispersion error, total error decreases as $k$ increases. This is because of the numerical effects of dispersion and dissipation prevalent in numerical methods. In Figs. (2) and (4), we are comparing the dissipation only. However, using the notion of composite schemes [5], the dispersion in a scheme is reduced by the inherent dissipation. A composite scheme made up of Lax-Wendroff and Lax-Friedrichs has better dispersive and dissipative properties than either Lax-Wendroff or LaxFriedrichs. Hence to understand the efficiency of a scheme, it is better to look at both the dispersive and dissipative properties.

The Unconditionally Positive Finite Difference Scheme at $h=0.1$ is consistent only at $k=0.001$. From Tables (1) to (3), the Non-Standard Finite Difference scheme at $k=\frac{0.85}{180}, h=0.1$ is the best scheme. The Upwind Forward Euler and NSFD scheme are best when $k=\frac{0.85}{180}$. Fig. (7) shows the exact and numerical solutions by Upwind Forward Euler and NSFD schemes with $k=\frac{0.85}{180}$ and Unconditionally Positive Finite Difference with $k=0.001$. The Unconditionally Positive Definite scheme gives best results when $k=0.005, h=0.1$ though it is not consistent for these values of $k$ and $h$. The scheme is still stable at $k=0.005$ unlike the Upwind Forward Euler and Non-Standard Finite Difference Scheme. Also the errors increase as $k$ is increased at a fixed $h(h=0.1)$ in the case of the Unconditionally Positive Finite Difference scheme.

\begin{tabular}{|c|c|c|c|c|c|}
\hline \hline$k$ & No of time steps & $L_{1}$ error & Dissipation Error & Dispersion Error & Total Error \\
\hline \hline 0.001 & 851 & $3.4013 \times 10^{-2}$ & $1.9150 \times 10^{-5}$ & $1.3679 \times 10^{-5}$ & $3.2829 \times 10^{-5}$ \\
\hline$\frac{0.85}{527}$ & 528 & $3.3810 \times 10^{-2}$ & $1.8923 \times 10^{-5}$ & $1.3519 \times 10^{-5}$ & $3.2442 \times 10^{-5}$ \\
\hline 0.002 & 426 & $3.3782 \times 10^{-2}$ & $1.8780 \times 10^{-5}$ & $1.3418 \times 10^{-5}$ & $3.2198 \times 10^{-5}$ \\
\hline$\frac{0.85}{212}$ & 213 & $3.3017 \times 10^{-2}$ & $1.8047 \times 10^{-5}$ & $1.2900 \times 10^{-5}$ & $3.0947 \times 10^{-5}$ \\
\hline$\frac{0.85}{180}$ & 181 & $3.2881 \times 10^{-2}$ & $1.7791 \times 10^{-5}$ & $1.2118 \times 10^{-5}$ & $3.0210 \times 10^{-5}$ \\
\hline \hline
\end{tabular}

Table 1: Errors for Upwind Forward Euler Finite Difference Scheme for Problem 1.

\begin{tabular}{|c|c|c|c|c|c|}
\hline \hline$k$ & No of time steps & $L_{1}$ error & Dissipation Error & Dispersion Error & Total Error \\
\hline \hline 0.001 & 851 & $3.3340 \times 10^{-2}$ & $1.8402 \times 10^{-5}$ & $1.3146 \times 10^{-5}$ & $3.1549 \times 10^{-5}$ \\
\hline$\frac{0.85}{527}$ & 528 & $3.2724 \times 10^{-2}$ & $1.7732 \times 10^{-5}$ & $1.2670 \times 10^{-5}$ & $3.0401 \times 10^{-5}$ \\
\hline 0.002 & 426 & $3.2336 \times 10^{-2}$ & $1.7315 \times 10^{-5}$ & $1.2373 \times 10^{-5}$ & $2.9688 \times 10^{-5}$ \\
\hline$\frac{0.85}{212}$ & 213 & $3.0323 \times 10^{-2}$ & $1.5230 \times 10^{-5}$ & $1.0891 \times 10^{-5}$ & $2.4910 \times 10^{-5}$ \\
\hline$\frac{0.85}{180}$ & 181 & $2.9609 \times 10^{-2}$ & $1.4523 \times 10^{-5}$ & $1.0387 \times 10^{-5}$ & $2.4910 \times 10^{-5}$ \\
\hline \hline
\end{tabular}

Table 2: Errors for Non-Standard Finite Difference Scheme for Problem 1.

\begin{tabular}{|c|c|c|c|c|c|}
\hline \hline$k$ & No of time Steps & $L_{1}$ error & Dissipation Error & Dispersion Error & Total Error \\
\hline \hline 0.0005 & 1701 & $3.2800 \times 10^{-2}$ & $1.8155 \times 10^{-5}$ & $1.2932 \times 10^{-5}$ & $3.1086 \times 10^{-5}$ \\
\hline 0.001 & 851 & $9.2257 \times 10^{-2}$ & $1.4700 \times 10^{-4}$ & $1.0500 \times 10^{-4}$ & $2.5200 \times 10^{-4}$ \\
\hline 0.002 & 426 & $1.9340 \times 10^{-1}$ & $6.7025 \times 10^{-4}$ & $4.7903 \times 10^{-4}$ & $1.1000 \times 10^{-3}$ \\
\hline 0.005 & 171 & $4.0430 \times 10^{-1}$ & $3.2000 \times 10^{-3}$ & $2.2000 \times 10^{-3}$ & $5.4000 \times 10^{-3}$ \\
\hline \hline
\end{tabular}

Table 3: Errors for Unconditionally Positive Finite Difference scheme for Problem 1. 


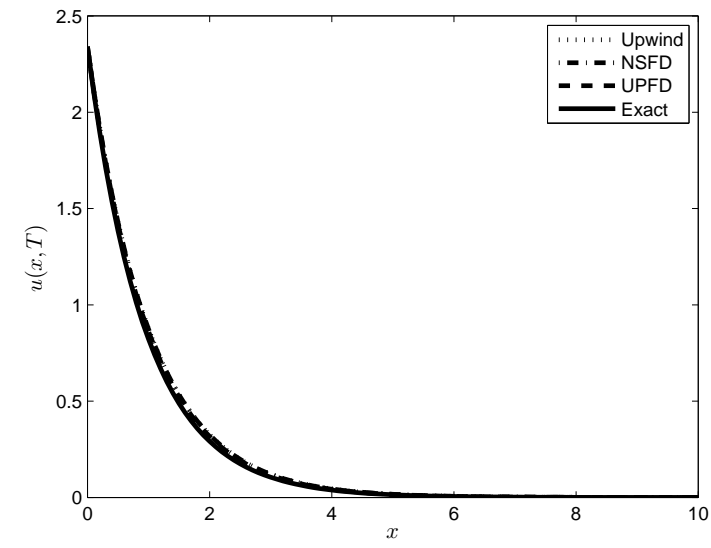

(a) Upwind forward Euler, NSFD, UPFD and exact solution compared $(k=0.001)$.

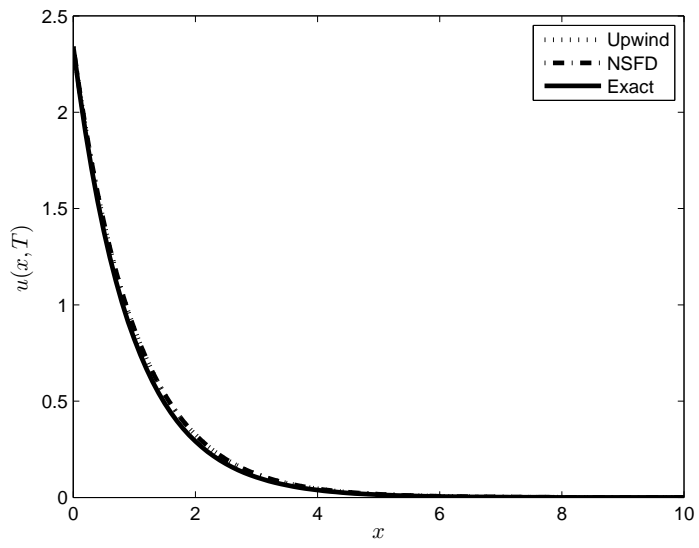

(b) Upwind forward Euler, NSFD and exact solution compared $\left(k=\frac{0.85}{180}\right)$.

Figure 7: Plots of numerical solution from Upwind Forward Euler, Non-Standard Finite Difference and Unconditionally Positive Finite Difference schemes at time, $T=0.85$.

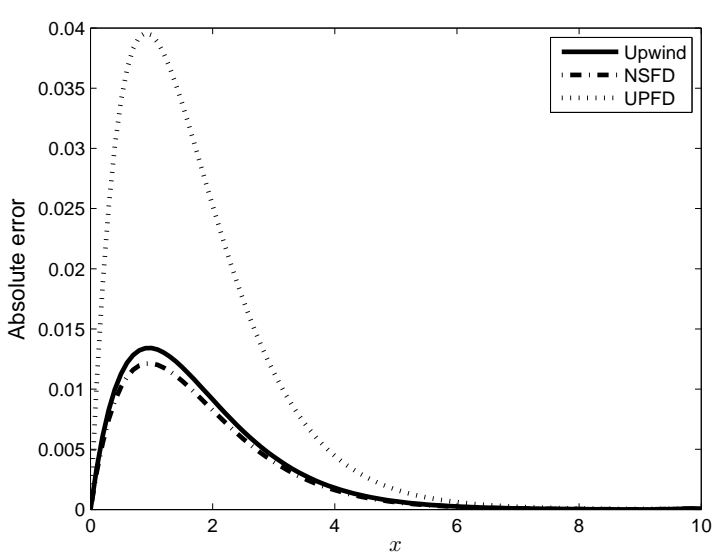

(a) $k=0.001$

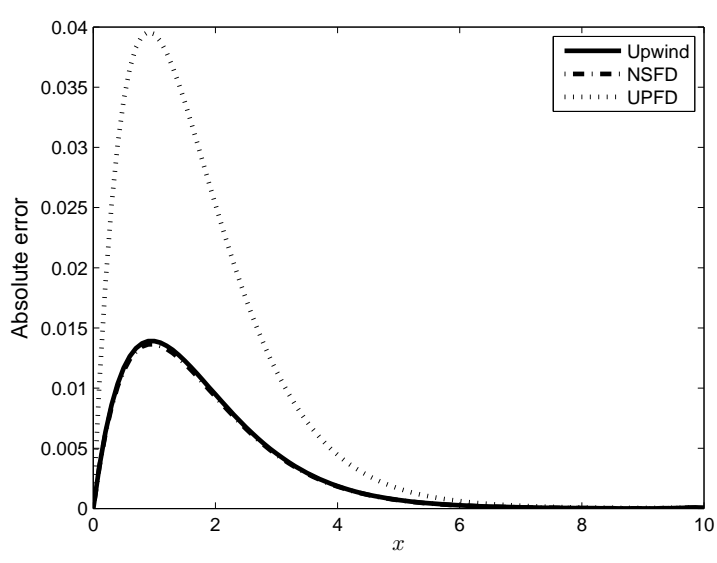

(b) $k=\frac{0.85}{180}$

Figure 8: Plots of absolute errors from Upwind Forward Euler, Non-Standard Finite Difference and Unconditionally Positive Finite Difference schemes at time, $T=0.85$. 


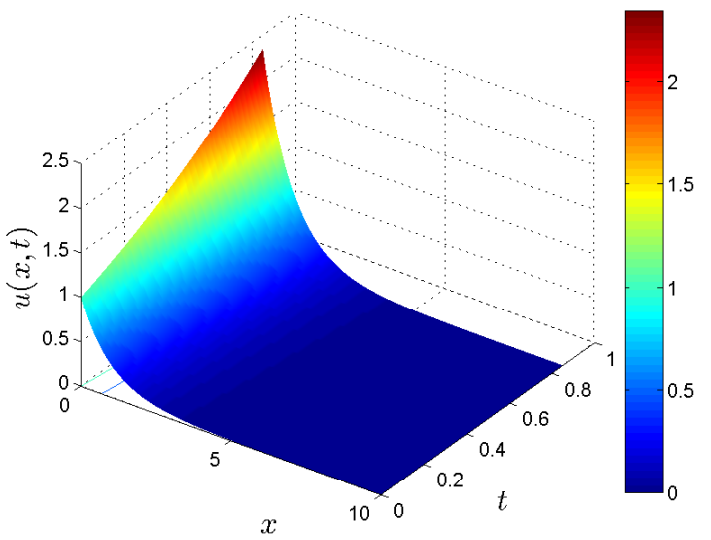

(a) Upwind Forward Euler scheme with $k=\frac{0.85}{180}$.

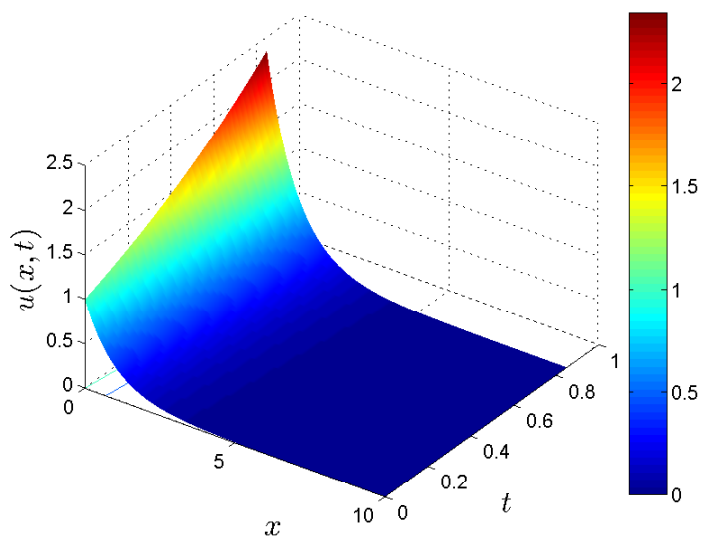

(b) NSFD scheme with $k=\frac{0.85}{180}$.

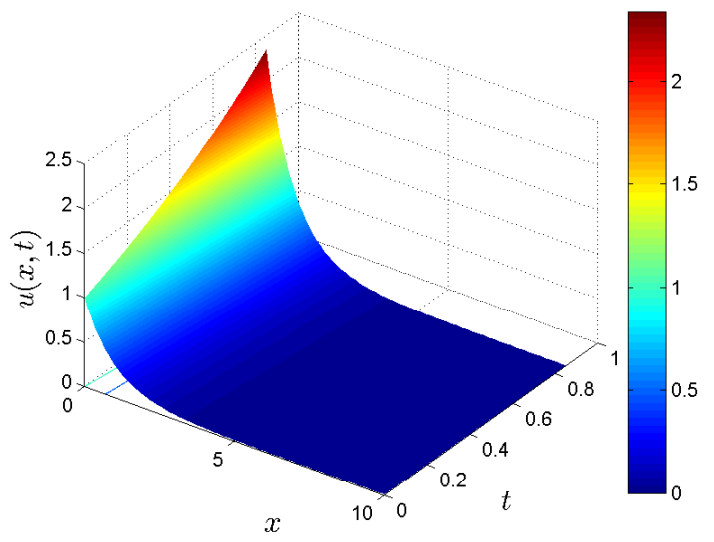

(c) UPFD scheme with $k=0.001$.

Figure 9: Numerical solutions using Upwind Forward Euler, NSFD and UPFD schemes plotted vs $t \in[0,0.85]$ vs $x \in[0,10]$.

\section{$6 \quad$ Numerical Solution of $u_{t}-\left(D u_{x}\right)_{x}=r u(1-u)$}

In this section, we use the three methods discussed in section 5 in order to solve $u_{t}-\left(D u_{x}\right)_{x}=r u(1-u)$, subject to specified initial and boundary conditions.

\subsection{Upwind Forward Euler Finite Difference Scheme (UPFD)}

The scheme when used to discretise Eq.(3) is given by [6]

$$
\frac{u_{m}^{n+1}-u_{m}^{n}}{k}-D\left(\frac{u_{m+1}^{n}-2 u_{m}^{n}+u_{m-1}^{n}}{h^{2}}\right)=r u_{m}^{n}\left(1-u_{m}^{n}\right),
$$

which simplifies to

$$
u_{m}^{n+1}=(1-2 \beta) u_{m}^{n}+k r\left(1-u_{m}^{n}\right) u_{m}^{n}+\beta\left(u_{m+1}^{n}+u_{m-1}^{n}\right),
$$


where $\beta=\frac{D k}{h^{2}}$.

\section{Stability}

The Fourier series analysis can only be applied directly to linear problems with constant coefficients. Since our problem is non-linear, the method will have a drawback. To overcome this drawback, we linearise the problem and freeze the coefficients[8]. The Fourier series analysis can then be applied to determine the region of stability thereafter.

Taha and Ablowitz [16] have obtained the stability of a scheme proposed by Zabusky and Kruskal [17] for the KdV equation using the method of freezing coefficients. The scheme derived by Zabusky and Kruskal for the KdV equation, $u_{t}+6 u u_{x}+u_{x x x}=0$ is

$$
u_{m}^{n+1}=u_{m}^{n-1}-\frac{2 k}{h}\left(u_{m+1}^{n}+u_{m}^{n}+u_{m-1}^{n}\right)\left(u_{m+1}^{n}-u_{m-1}^{n}\right)-\frac{k}{h^{3}}\left(u_{m+2}^{n}-2 u_{m+1}^{n}+2 u_{m-1}^{n}-u_{m-2}^{n}\right) .
$$

Using the method of freezing coefficients and Fourier analysis, they write $u u_{x}$ as $u_{\max } u_{x}$ and substitute the ansatz $u_{m}^{n}=\xi^{n} \exp (\operatorname{Im} \omega)$ where $\omega$ is the phase angle.

The amplification polynomial is given by

$$
\xi=\xi^{-1}-\frac{2 k}{h} u_{\max }(2 I \sin (\omega))-\frac{k}{h^{3}}(\exp (2 I \omega)-2 \exp (I \omega)+2 \exp (-I \omega)-\exp (-2 I \omega)),
$$

and the region of stability is obtained as

$$
\frac{k}{h} \leq \frac{2}{3 \sqrt{3}\left(2 u_{\max }-\frac{1}{h^{2}}\right)} .
$$

We use the same approach used by Taha and Ablowitz to obtain the stability of schemes discretising $u_{t}-\left(D u_{x}\right)_{x}=r u(1-u)$.

Using Eq.(34), we have

$$
u_{m}^{n+1}=u_{m}^{n}+\frac{D k}{h^{2}}\left(u_{m+1}^{n}-2 u_{m}^{n}+u_{m-1}^{n}\right)+k r u_{m}^{n}-\left(k r u_{m}^{n}\right) u_{\max },
$$

where $u_{\max }$ is the frozen coefficient.

Applying Fourier series analysis to Eq.(35), we obtain the amplification factor as

$$
\xi=1+\frac{2 D k}{h^{2}}(\cos \omega-1)+k r\left(1-u_{\max }\right) .
$$

Using the trigonometric identity, $1-\cos \omega \equiv 2 \sin ^{2}\left(\frac{\omega}{2}\right)$, we obtain

$$
\xi=1-\frac{4 D k}{h^{2}} \sin ^{2}\left(\frac{\omega}{2}\right)+k r\left(1-u_{\max }\right) .
$$

When $\omega=0$, we must have $\xi=1$. Hence we choose $u_{\max }=1$.0. Also, we choose $D=0.0002, h=0.01$ and $r=0.05$. We thus have

$$
\xi=1-8 k \sin ^{2}\left(\frac{\omega}{2}\right),
$$

where $-\pi \leq \omega \leq \pi$.

and for stability, we have

$$
0<k \leq 0.25 \text {. }
$$




\section{Consistency}

To obtain the truncation errors for the Upwind Forward Euler finite difference scheme, we need to first determine the Taylor expansion of the terms in Eq.(34) about the grid point $(m, n)$. Substituting Eqs. (12)-(14) in (34) and simplifying, we have

$$
\frac{\partial u}{\partial t}-D \frac{\partial^{2} u}{\partial x^{2}}=r u(1-u)-\frac{k}{2} \frac{\partial^{2} u}{\partial t^{2}}-\frac{k^{2}}{6} \frac{\partial^{3} u}{\partial t^{3}}+\cdots
$$

As $k \rightarrow 0$, Eq. (40) reduces to Eq.(3) and so the Upwind Forward Euler finite difference scheme is consistent.

Fig. (10) shows the variation of the modulus of amplification factor for the Upwind Forward Euler scheme vs phase angle at $h=0.01$, with some different values of $k$. As $k$ increases, for a given angle, the numerical dissipation increases.

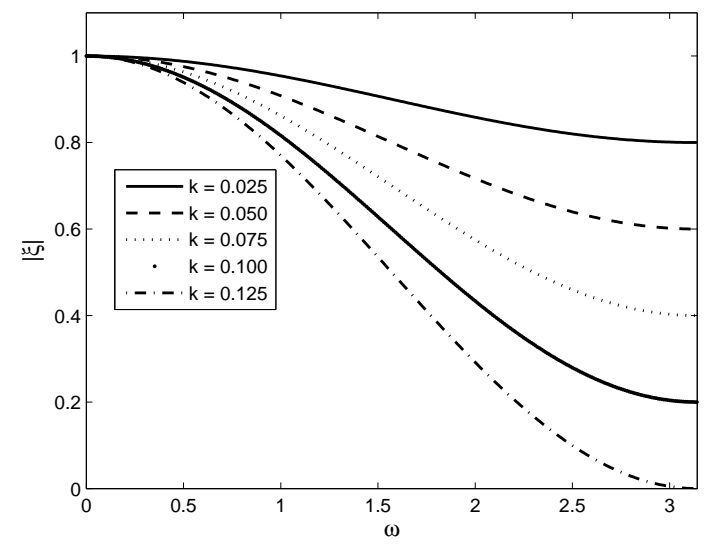

(a)

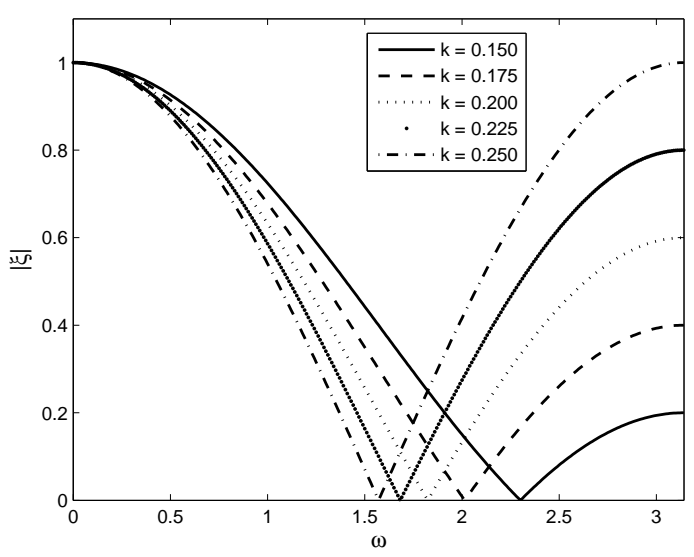

(b)

Figure 10: Plot of modulus of amplification factor for Upwind forward Euler scheme for some values of $k$, with $h=0.01$, when $u_{\max }=1.0$.

\section{Spectral Analysis}

To study the dispersion of numerical schemes discretising the partial differential equation,

$$
\frac{\partial u}{\partial t}=2 \alpha u \frac{\partial u}{\partial x}+\gamma \frac{\partial u}{\partial x}+\nu \frac{\partial^{3} u}{\partial x^{3}},
$$

Asher and McLachlan [4] considered the linearised version of Eq.(41) which is

$$
\frac{\partial u}{\partial t}=\gamma \frac{\partial u}{\partial x}+\nu \frac{\partial^{3} u}{\partial x^{3}} .
$$

We use the same approach that they used. The linearised form of Eq.(3) is given by

$$
\frac{\partial u}{\partial t}-D \frac{\partial^{2} u}{\partial x^{2}}=r u
$$

We then consider the perturbation

$$
u(x, t)=\mathrm{e}^{\alpha t} \mathrm{e}^{I \theta x} .
$$


Substituting Eq.(44) in (43) and solving for the dispersion relation, $\alpha$, we obtain

$$
\alpha=r-D \theta^{2} .
$$

Since the dispersion relation does not have an imaginary part, the relative phase error cannot be computed using the formula in Eq.(21).

\subsection{Non-Standard Finite Difference Scheme}

Eq. (3) is approximated by [11]

$$
\frac{u_{m}^{n+1}-u_{m}^{n}}{k}-D\left(\frac{u_{m+1}^{n}-2 u_{m}^{n}+u_{m-1}^{n}}{h^{2}}\right)=r u_{m}^{n}-r\left(\frac{u_{m+1}^{n}+u_{m}^{n}+u_{m-1}^{n}}{3}\right) u_{m}^{n+1},
$$

which simplifies to

$$
u_{m}^{n+1}=\frac{(1+k r-2 \beta) u_{m}^{n}+\beta\left(u_{m+1}^{n}+u_{m-1}^{n}\right)}{1+k r\left(\frac{u_{m+1}^{n}+u_{m}^{n}+u_{m-1}^{n}}{3}\right)},
$$

where $\beta=\frac{D k}{h^{2}}$.

\section{Stability}

Applying the Fourier series analysis and the method of freezing coefficients to Eq.(46), we obtain the amplification factor for the NSFD scheme as

$$
\xi=\frac{1+k r+\frac{2 D k}{h^{2}}(\cos \omega-1)}{1+k r u_{\max }} .
$$

Using the trigonometric identity, $1-\cos \omega \equiv 2 \sin ^{2}\left(\frac{\omega}{2}\right)$ we have

$$
\xi=\frac{1+k r-\frac{4 D k}{h^{2}} \sin ^{2}\left(\frac{\omega}{2}\right)}{1+k r u_{\max }} .
$$

When $\omega=0$, we must have $\xi=1$, hence $u_{\max }$ is chosen as 1.0.

Setting $D=0.0002, r=0.05, h=0.01$ and $u_{\max }=1.0$, the amplification factor is given as

$$
\xi=\frac{1+0.05 k-8 k \sin ^{2}\left(\frac{\omega}{2}\right)}{1+0.05 k} .
$$

For stability, we have

$$
-1 \leq \frac{1+0.05 k-8 k \sin ^{2}\left(\frac{\omega}{2}\right)}{1+0.05 k} \leq 1
$$

Since this condition must hold for every phase angle, $\omega \in[-\pi, \pi]$ we have $8 k \leq 2+0.1 k$ which gives $k \leq 0.253$. Hence the region of stability is given by

$$
0<k \leq 0.253 \text {. }
$$

We note that in [6], they have used $k=0.26$ when $h$ is chosen as 0.01 . However, from our stability analysis, we observe that the Upwind Forward Euler and NSFD schemes are not stable for $k>0.25$ and $k>0.253$ respectively. 


\section{Consistency}

To obtain the truncation errors of the NSFD scheme, we need to first determine the Taylor expansion of the terms in Eq. (46) about $(m, n)$. Substituting Eqs.(12)-(14) in (46) and simplifying, we have

$$
\begin{aligned}
\frac{\partial u}{\partial t}-D \frac{\partial^{2} u}{\partial x^{2}} & =r u(1-u)-\frac{r h}{3} u^{2}-\left(r k u+\frac{r k h}{3} u\right) \frac{\partial u}{\partial t}-\left(\frac{k}{2}+\frac{k^{2} r}{2} u+\frac{r k^{2} h}{6} u\right) \frac{\partial^{2} u}{\partial t^{2}} \\
& -\left(\frac{k^{2}}{6}+\frac{r k^{3}}{6} u+\frac{r h k^{3}}{18} u\right) \frac{\partial^{3} u}{\partial t^{3}}+\cdots
\end{aligned}
$$

As $k \rightarrow 0$ and $h \rightarrow 0$, Eq.(49) reduces to Eq. (3) and hence the NSFD scheme is consistent.

Fig.(11) shows the modulus of amplification factor vs phase angle for the Non-Standard Finite Difference scheme for some values of $k$, with $h=0.01$. It is seen that the scheme is stable for $0<k \leq 0.253$.

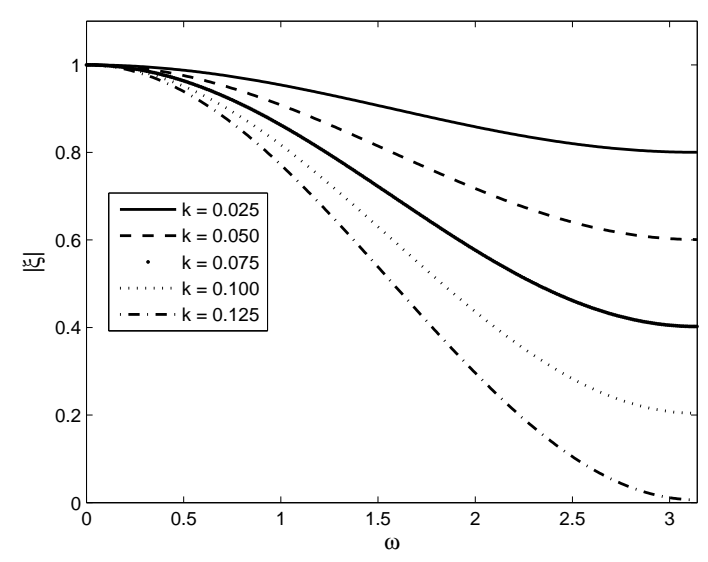

(a)

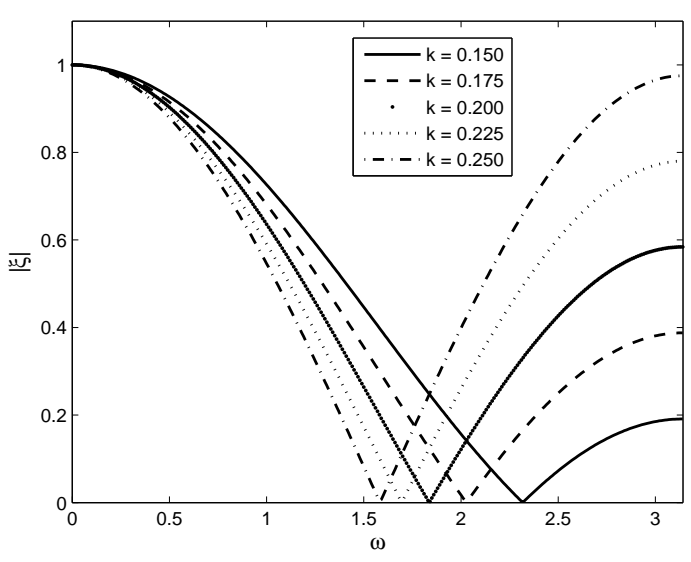

(b)

Figure 11: Plot of modulus of amplification factor vs $\omega \in[0, \pi]$ for Non-Standard Finite Difference scheme for some values of $k$, with $h=0.01$, with $u_{\max }=1.0$.

\subsection{Unconditionally Positive Finite Difference Scheme}

The UPFD scheme when used to approximate Eq.(3) is given by [6]

$$
\frac{u_{m}^{n+1}-u_{m}^{n}}{k}-D\left(\frac{u_{m+1}^{n}-2 u_{m}^{n+1}+u_{m-1}^{n}}{h^{2}}\right)=r u_{m}^{n}-r u_{m}^{n} u_{m}^{n+1},
$$

which simplifies to

$$
u_{m}^{n+1}=\frac{(1+k r) u_{m}^{n}+\beta\left(u_{m+1}^{n}+u_{m-1}^{n}\right)}{1+2 \beta+k r u_{m}^{n}}, \quad \text { where } \beta=\frac{D k}{h^{2}} .
$$

\section{Stability}

Applying the Fourier series analysis and method of freezing coefficients to Eq.(50), we obtain the amplification factor for the Unconditionally Positive Finite Difference scheme given as

$$
\xi=\frac{1+k r+2 \beta \cos \omega}{1+2 \beta+k r u_{\max }} .
$$


where $\beta=\frac{D k}{h^{2}}$.

When $\omega=0$, we must have $\xi=1$. Hence $u_{\max }=1.0$

Setting $D=0.0002, r=0.05, h=0.01$ and $u_{\max }=1.0$, we have amplification factor given by

$$
\xi=\frac{1+4 k \cos \omega+0.05 k}{1+4.05 k} .
$$

The scheme is unconditionally stable.

We note that the UPFD scheme is unconditionally stable while the NSFD and Upwind Forward Euler methods have almost the same region of stability.

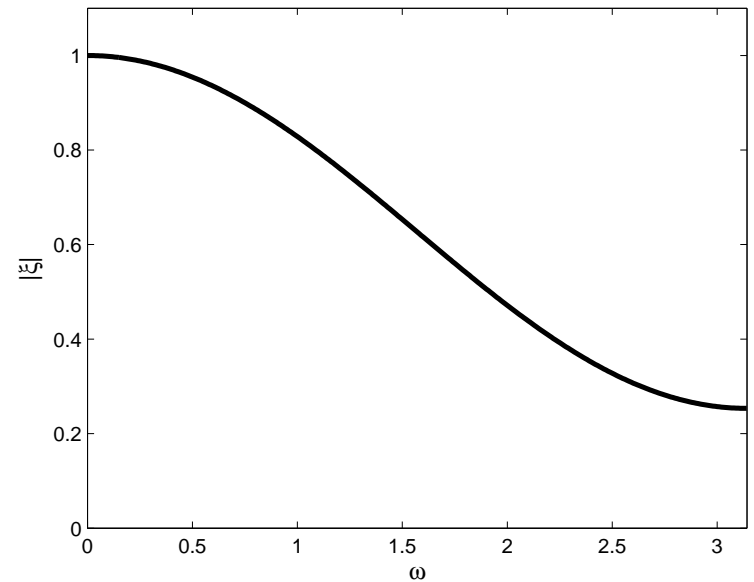

Figure 12: Plots of modulus of amplification factor for Unconditionally Positive Finite Difference scheme for $k=h^{3}$, with $h=0.01$.

\subsection{Consistency}

To obtain the truncation errors for the Unconditionally Positive Finite difference scheme we need to first determine the Taylor expansion of the terms in Eq.(50) about $(m, n)$. Substituting Eqs.(12)-(14) in (50) and simplifying we have

$$
\begin{aligned}
\frac{\partial u}{\partial t}-D \frac{\partial^{2} u}{\partial x^{2}} & =r u(1-u)-\left(k r u+\frac{2 D k}{h^{2}}\right) \frac{\partial u}{\partial t}-\left(\frac{k}{2}+\frac{D k^{2}}{h^{2}}+\frac{k^{2} r}{2} u\right) \frac{\partial^{2} u}{\partial t^{2}} \\
& -\left(\frac{k^{2}}{6}+\frac{D k^{3}}{3 h^{2}}-\frac{k^{3} r}{6} u\right) \frac{\partial^{3} u}{\partial t^{3}}+\cdots
\end{aligned}
$$

To ensure that the UPFD scheme is consistent, we choose the time step that depends on the spatial step such that the truncation error reduces to zero. For this reason we let $k=h^{3}$. Hence, we have

$$
\begin{aligned}
\frac{\partial u}{\partial t}-D \frac{\partial^{2} u}{\partial x^{2}} & =r u(1-u)-\left(h^{3} r u+2 D h\right) \frac{\partial u}{\partial t}-\frac{h^{3}}{2}\left(1+2 D h+r h^{3} u\right) \frac{\partial^{2} u}{\partial t^{2}} \\
& -\frac{h^{6}}{6}\left(1+2 D h+r h^{3} u\right) \frac{\partial^{3} u}{\partial t^{3}}+\cdots
\end{aligned}
$$

As $h \rightarrow 0$, Eq. (53) reduces to Eq.(3) and so the scheme is consistent. 


\section{Numerical Results for Experiment 2}

We tabulate the error rate with respect to $L_{1}$ norm, dissipation, dispersion and total error in Tables (4) to Table (6) for the three numerical schemes. Based on Tables (4) to (6), we observe that dispersion error is greater than dissipation error at a given value of $k$ for all the three schemes. As $k$ increases, dissipation, dispersion error and total errors are not very much affected.

In the case of Upwind Forward Euler finite difference scheme, $E_{\text {num }}$ is smallest when $k=0.125$ and the total error is least when $k=0.050$. In the case of Non-Standard Finite Difference scheme, errors either increase or decrease as $k$ is increased i.e there is no general behaviour. The NSFD scheme performs best at $k=0.125$ with $E_{\text {num }}$ and total error being $5.5968 \times 10^{-2}$ and $6.8480 \times 10^{-3}$. The values for $E_{\text {num }}$ and Total error are $5.6085 \times 10^{-2}$ and $6.8630 \times 10^{-3}$ respectively for the Unconditionally Positive Finite Difference scheme when it is consistent with $k=10^{-6}$ and $h=0.01$ and consequently it took several hours to obtain the results from the code in Matlab using this scheme.

\begin{tabular}{|c|c|c|c|c|c|}
\hline \hline$k$ & No of time steps & $L_{1}$ error & Dissipation Error & Dispersion Error & Total Error \\
\hline \hline 0.025 & 521 & $5.6103 \times 10^{-2}$ & $2.6710 \times 10^{-3}$ & $4.1980 \times 10^{-3}$ & $6.8700 \times 10^{-3}$ \\
\hline 0.050 & 261 & $5.6146 \times 10^{-2}$ & $2.6800 \times 10^{-3}$ & $4.2040 \times 10^{-3}$ & $6.8440 \times 10^{-3}$ \\
\hline 0.075 & 174 & $5.6072 \times 10^{-2}$ & $2.6750 \times 10^{-3}$ & $4.1970 \times 10^{-3}$ & $6.8720 \times 10^{-3}$ \\
\hline 0.100 & 130 & $5.6116 \times 10^{-2}$ & $2.6750 \times 10^{-3}$ & $4.2070 \times 10^{-3}$ & $6.8820 \times 10^{-3}$ \\
\hline 0.125 & 105 & $5.6042 \times 10^{-2}$ & $2.6620 \times 10^{-3}$ & $4.2030 \times 10^{-3}$ & $6.8650 \times 10^{-3}$ \\
\hline 0.150 & 88 & $5.6554 \times 10^{-2}$ & $2.7430 \times 10^{-3}$ & $4.2550 \times 10^{-3}$ & $6.9980 \times 10^{-3}$ \\
\hline 0.175 & 75 & $5.6070 \times 10^{-2}$ & $2.6860 \times 10^{-3}$ & $4.2030 \times 10^{-3}$ & $6.8880 \times 10^{-3}$ \\
\hline 0.200 & 66 & $5.6289 \times 10^{-2}$ & $2.7110 \times 10^{-3}$ & $4.2310 \times 10^{-3}$ & $6.9410 \times 10^{-3}$ \\
\hline 0.225 & 59 & $5.6509 \times 10^{-2}$ & $2.7360 \times 10^{-3}$ & $4.2590 \times 10^{-3}$ & $6.9940 \times 10^{-3}$ \\
\hline 0.250 & 53 & $5.6509 \times 10^{-2}$ & $2.7360 \times 10^{-3}$ & $4.2590 \times 10^{-3}$ & $6.9940 \times 10^{-3}$ \\
\hline \hline
\end{tabular}

Table 4: Errors for Upwind Forward Euler Finite Difference scheme for Problem 2.

\begin{tabular}{|c|c|c|c|c|c|}
\hline \hline$k$ & No of time steps & $L_{1}$ error & Dissipation Error & Dispersion Error & Total Error \\
\hline \hline 0.025 & 521 & $5.6087 \times 10^{-2}$ & $2.7000 \times 10^{-3}$ & $4.1870 \times 10^{-3}$ & $6.8670 \times 10^{-3}$ \\
\hline 0.050 & 261 & $5.6116 \times 10^{-2}$ & $2.6870 \times 10^{-3}$ & $4.1910 \times 10^{-3}$ & $6.8780 \times 10^{-3}$ \\
\hline 0.075 & 174 & $5.6027 \times 10^{-2}$ & $2.6800 \times 10^{-3}$ & $4.1820 \times 10^{-3}$ & $6.8620 \times 10^{-3}$ \\
\hline 0.100 & 130 & $5.6056 \times 10^{-2}$ & $2.6780 \times 10^{-3}$ & $4.1900 \times 10^{-3}$ & $6.8680 \times 10^{-3}$ \\
\hline 0.125 & 105 & $5.5968 \times 10^{-2}$ & $2.6630 \times 10^{-3}$ & $4.1850 \times 10^{-3}$ & $6.8480 \times 10^{-3}$ \\
\hline 0.150 & 88 & $5.6465 \times 10^{-2}$ & $2.7420 \times 10^{-3}$ & $4.2340 \times 10^{-3}$ & $6.9760 \times 10^{-3}$ \\
\hline 0.175 & 75 & $5.5957 \times 10^{-2}$ & $2.6830 \times 10^{-3}$ & $4.1800 \times 10^{-3}$ & $6.8630 \times 10^{-3}$ \\
\hline 0.200 & 66 & $5.6171 \times 10^{-2}$ & $2.7060 \times 10^{-3}$ & $4.2060 \times 10^{-3}$ & $6.9120 \times 10^{-3}$ \\
\hline 0.225 & 59 & $5.6375 \times 10^{-2}$ & $2.7290 \times 10^{-3}$ & $4.2320 \times 10^{-3}$ & $6.9610 \times 10^{-3}$ \\
\hline 0.250 & 53 & $5.6112 \times 10^{-2}$ & $2.6970 \times 10^{-3}$ & $4.2050 \times 10^{-3}$ & $6.9020 \times 10^{-3}$ \\
\hline \hline
\end{tabular}

Table 5: Errors for Non-Standard Finite Difference scheme for Problem 2.

\begin{tabular}{|c|c|c|c|c|c|}
\hline \hline$k$ & No of time Steps & $L_{1}$ error & Dissipation Error & Dispersion Error & Total Error \\
\hline \hline $1.0 \times 10^{-6}$ & $13 \times 10^{6}$ & $5.6085 \times 10^{-2}$ & $2.6690 \times 10^{-3}$ & $4.1930 \times 10^{-3}$ & $6.8630 \times 10^{-3}$ \\
\hline \hline
\end{tabular}

Table 6: Errors for Unconditionally Positive Finite Difference scheme for Problem 2. 


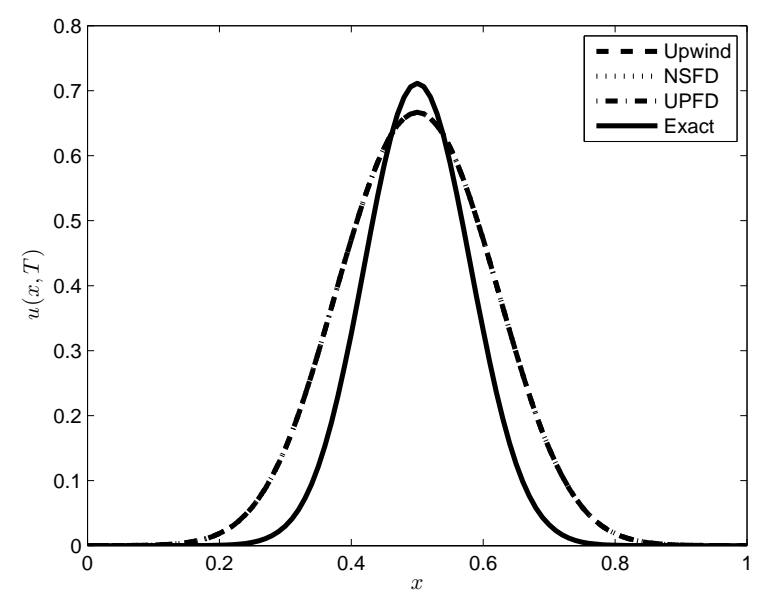

Figure 13: Plots of numerical solution from Upwind Forward Euler $(k=0.125)$, NSFD $(k=0.125)$, UPFD $\left(k=1.0 \times 10^{-6}\right)$ and exact solution at time, $T=13$.

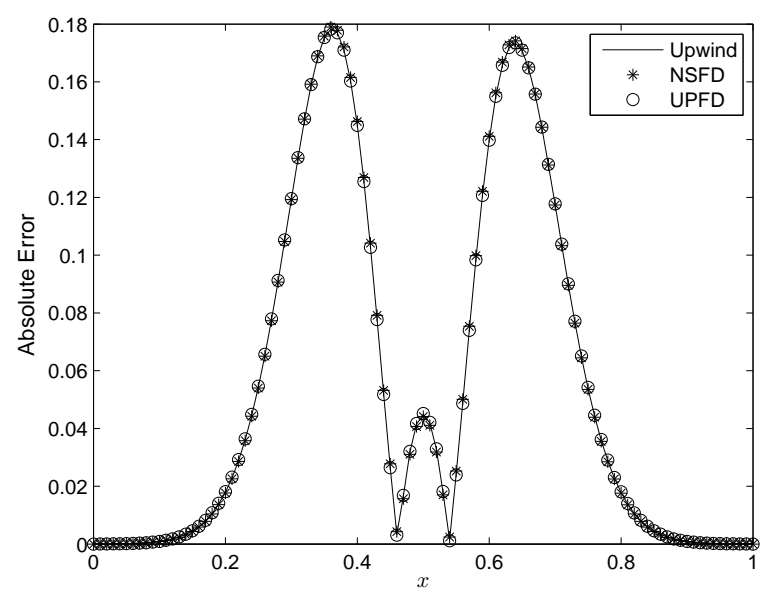

Figure 14: Plots of absolute error for the Upwind forward Euler $(k=0.125), \operatorname{NSFD}(k=0.125)$ and UPFD $\left(k=1.0 \times 10^{-6}\right)$ schemes with $h=0.01$. 


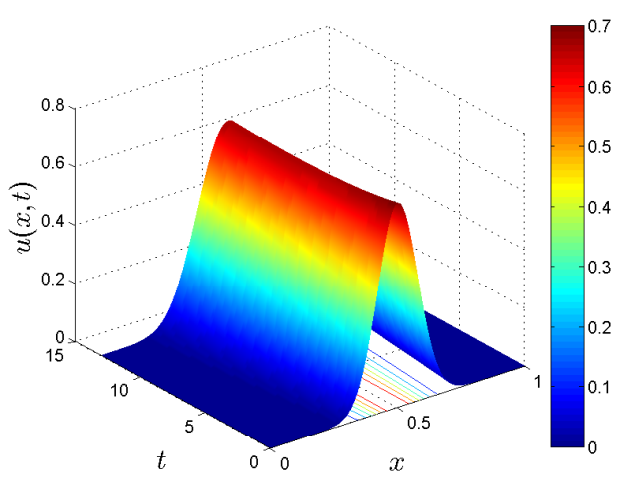

(a) Upwind Forward Euler scheme with $k=$ 0.125

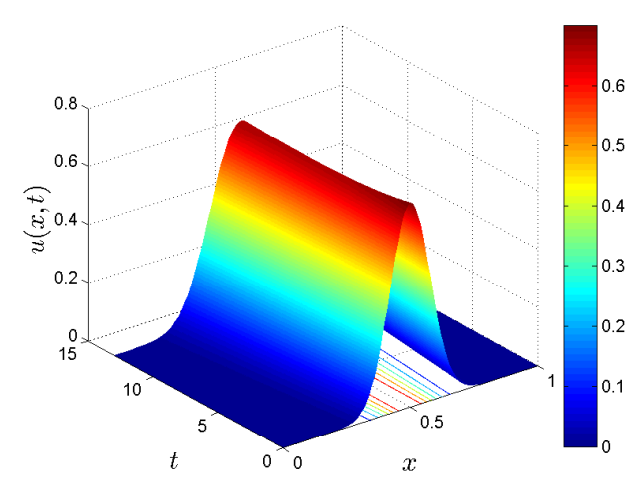

(b) NSFD scheme with $k=0.250$

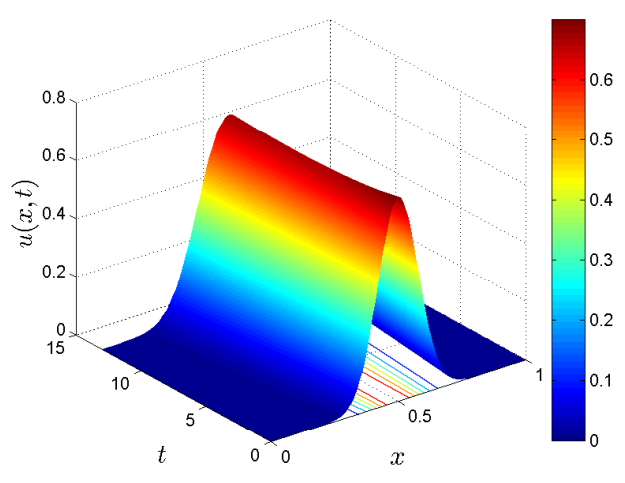

(c) UPFD scheme with $k=1.0 \times 10^{-6}$

Figure 15: Numerical solutions using Upwind Forward Euler, NSFD and UPFD schemes plotted vs time, $t \in[0,13]$ vs space, $x \in[0,1]$.

\section{Conclusion}

In this paper, three finite difference schemes have been used to solve a linear and a non-linear advectiondiffusion-reaction equation, both with constant convective velocity and diffusion. The errors from the Non-Standard Finite Difference scheme are less than that for the Upwind Forward Euler scheme and Unconditionally Positive Finite Difference scheme for the first experiment. For the second experiment which models a non-linear diffusion reaction, the errors for the three schemes are not very different at a given value of $h$ and $k$.

The Unconditionally Positive Finite Difference scheme suffers a drawback when it comes to consistency. When spatial step becomes smaller, the computations requires more CPU time and power. This drawback was experienced in experiment 2 where the number of time steps required was $1.3 \times 10^{7}$.

In the two cases considered, the velocity was constant, i.e we have a steady fluid flow. However we can also have transient fluid flow where the velocity can be dependent on time. The analysis of dispersive and dissipative properties of numerical methods in the case of transient fluid flow can be very complicated as we have additional parameters involved. One way to handle transient fluid flow problems is to consider higher order Nonstandard finite difference schemes such as those used in Anguelov et al. [1] as first order methods cannot handle such problems. 


\section{Acknowledgements}

The authors acknowledge the financial support of DST/NRF SARChI chair in Mathematical Models and Methods in Bioengineering and BioSciences of the University of Pretoria and would like to thank the three anonymous reviewers for their very constructive comments and suggestions which have helped to improve the paper considerably. Dr Appadu is also grateful to the Research Development Programme of the University of Pretoria for funding.

\section{References}

[1] R. Anguelov, P. Kama and J. M. S. Lubuma, On non-standard finite difference models of reactiondiffusion equations, Journal of Computational and Applied Mathematics 175, 2005, 11-29.

[2] A. R. Appadu, Numerical solution of the 1-D advection-diffusion equation using standard and nonstandard finite difference schemes, Journal of Applied Mathematics 15, 2013, 201-215.

[3] A. R. Appadu and M. Z. Dauhoo, The concept of minimized integrated exponential error for low dispersion and low dissipation, International Journal for Numerical Methods in Fluids 65, 2011, 578-601.

[4] M. Ascher and R. McLachlan, On Symplectic and Multisympletic Schemes for the KDV Equation, Journal of Scientific Computing 25, 2005.

[5] R. Liska and B. Wendroff, Composite schemes for Conservation Laws, SIAM Journal on Numerical Analysis 35, 6, 2250-2271.

[6] B. M. Chen-Charpentier and H. V. Kojouharov, Unconditionally positivity preserving scheme for advection-diffusion-reaction equations, Mathematical and Computer Modelling 57, 2013, 2177-1285.

[7] B. M. Chen-Charpentier and H. V. Kojouharov, Non-standard Numerical Methods Applied to Subsurface Biobarrier Formation Models in Porous Media, Bulletin of Mathematical Biology 61, 4, 1999, 779-798.

[8] D. R. Durran, Numerical Methods for Fluid Dynamics with Applications to Geophysics(Second Edition), Springer, 2010.

[9] J. M. Makungu, H. Haario and W. C. Mahera, A generalised 1-dimensional particle transport method for convection-diffusion-reaction model, African Mathematical Union and SpringerVerlag 23, 2011, 21-39.

[10] R. E. Mickens, Nonstandard finite difference schemes for reaction-diffusion equations, Numerical Methods for Partial Differential Equations 15, 1999, 201-215.

[11] R. E. Mickens, Nonstandard finite difference schemes for reaction-diffusion equations having linear advection, Numerical Methods for Partial Differential Equations 15, 4, 2000, 361-364.

[12] K. C. Patidar, On the use of nonstandard finite difference methods, Journal of Difference Equations and Application 11, 2005, 735-758.

[13] A. D. Polyanin and V. F. Zaitsev, Handbook of Nonlinear Partial Differential Equations, Chapman $\& \mathrm{Hall} / \mathrm{CRC}, 2004$. 
[14] T. W. Sheu, S. K. Wang and R. K. Lin, An Implicit Scheme for Solving the Convection-DiffusionReaction-Equation in Two Dimensions, Journal of Computational Physics 164, 2000, 123-142.

[15] J. C. Strikwerda, Finite Difference Schemes and Partial Differential Equations (Second Edition), Society for Industrial and Applied Mathematics, 2004.

[16] T. Taha and M. Ablowitz, Analytical and Numerical aspects of Certain Nonlinear Avolution Equations III. Numerical Korteweg-de Vries Equation, Journal of Computational Physics , 55, 231-253, 1984.

[17] N.J. Zabusky and M.D. Kruskal, Interaction of solitons in a collisionless plasma and the recurrence of initial states, Physical Review Letters , 15, 6, 240-243, 1965.

[18] L. C Takacs, A Two-Step Scheme for the Advection Equation with Minimized Dissipation and Dispersion Errors, Monthly Weather Review 113, 1985, 1050-1065. 